\title{
PRESERVATION OF PHASE-TYPE DISTRIBUTIONS UNDER POISSON SHOCK MODELS
}

\author{
M. MANOHARAN, \\ HARSHINDER SINGH AND \\ NEERAJ MISRA, Panjab University
}

\begin{abstract}
In this paper, we consider the life distribution $H(t)$ of a device subject to shocks governed by a finite mixture of homogeneous Poisson processes. It will be shown that if $\left(p_{k}\right)$, the probabilities that the device fails on the $k$ th shock, has a discrete phase-type (DPH) distribution, then $H(t)$ is continuous phase-type $(\mathrm{CPH})$. The relationship between the mean values of $\left(p_{k}\right)$ and $H(t)$ is established.
\end{abstract}

RELIABILITY; MIXED POISSON PROCESSES; SURVIVAL FUNCTION

AMS 1991 SUBJECT CLASSIFICATION: PRIMARY 62E99

\section{Introduction}

Suppose that a device is subjected to shocks occurring randomly over time according to a counting process $\mathcal{N}=\{N(t): t \geqq 0\}$. Let $p_{k}$ be the probability that the device fails on the $k$ th shock and $\bar{P}_{k}=\sum_{n=k+1}^{\infty} p_{n}$ be the probability that the device survives $k$ shocks, $k=0,1$, $2, \cdots$, where $1=\bar{P}_{0} \geqq \bar{P}_{1} \geqq \cdots$. Then it follows that the survival function $\bar{H}(t)$ of the device is given by

$$
\bar{H}(t)=\sum_{k=0}^{\infty} P\{N(t)=k\} \bar{P}_{k} .
$$

Shock models of this kind have been studied for instance by Esary et al. (1973) when $\mathcal{N}$ is a homogeneous Poisson process, by A-Hameed and Proschan (1973), (1975) when $\mathcal{N}$ is a non-homogeneous Poisson process or a birth process and by Block and Savits $(1978)$ when $\mathcal{N}$ is a more general counting process. In all these cases the authors prove that $\bar{H}(t)$ is increasing failure rate (IFR), increasing failure rate average (IFRA), decreasing mean residual life (DMRL), new better than used (NBU) or new better than used in expectation (NBUE) under suitable conditions on $\mathcal{N}$ if $\left(\bar{P}_{k}\right)_{k=0}^{\infty}$ has the corresponding discrete property. Klefsjö (1981) proves that $\bar{H}(t)$ is harmonic new better than used in expectation (HNBUE) if $\bar{P}_{k}$ has the discrete HNBUE property.

The aim of this paper is to prove the corresponding theorem for the phase-type distribution (see Neuts (1981)) under finite mixture of Poisson processes. In particular, it will be shown that phase-type distribution is preserved under the transformation described by (1.1) when $\mathcal{N}$ is a finite mixture of homogeneous Poisson processes. Also, the relationship between the mean values of $\left(p_{k}\right)$ and $H(t)$ is established.

\section{Phase-type distributions}

There are two parallel discussions of phase-type distributions; one corresponding to distributions on $[0, \infty)$ obtained from absorption times in continuous-parameter Markov

Received 5 November 1990; revision received 21 October 1991.

Postal address for all authors: Department of Statistics, Panjab University, Chandigarh-160014, India. 
chains, the other to distributions on the non-negative integers obtained from discreteparameter Markov chains. For details of this highly versatile class of probability distributions the reader is referred to Neuts (1981). Only relevant definitions and properties are mentioned here.

Definition 2.1. A distribution $F($.$) on [0, \infty)$ is a continuous phase-type $(\mathrm{CPH})$ distribution if it is that of the time until absorption in a finite-state Markov process with generator

$$
\boldsymbol{Q}=\left[\begin{array}{cc}
\boldsymbol{T} & \boldsymbol{T}^{0} \\
\mathbf{0} & 0
\end{array}\right]
$$

and initial probability vector $\left(\alpha, \alpha_{m+1}\right) . T=\left[T_{i j}\right]$ is a non-singular matrix of order $m$ and satisfies $T_{i i} \leqq 0$ for $1 \leqq i \leqq m, T_{i j} \geqq 0$ for $i \neq j$. Also $\boldsymbol{T e}_{m}+\boldsymbol{T}^{0}=0$ and $\alpha \boldsymbol{e}_{m}+\alpha_{m+1}=1$, where $\boldsymbol{e}_{m}$ denotes an $m$-column vector with all components equal to 1 . The distribution $F($.$) is given by$

$$
F(x)=1-\alpha \exp (T x) e_{m} \text { for } x \geqq 0
$$

and we say $F$ has the representation $(\boldsymbol{\alpha}, \boldsymbol{T})$. The distribution has a jump of magnitude $\alpha_{m+1}$ at the origin and the mean of the distribution is $\mu=-\alpha T^{-1} e_{m}$.

The analogous definition for the density $\left(p_{k}\right)$ on the non-negative integers is as follows. If $\left(p_{k}\right)$ is the density of the time until absorption in a finite-state Markov chain with stationary transition probability matrix given by

$$
\boldsymbol{P}=\left[\begin{array}{cc}
\boldsymbol{T} & \boldsymbol{T}^{0} \\
\mathbf{0} & 1
\end{array}\right]
$$

and initial probability vector $\left(\boldsymbol{\alpha}, \alpha_{m+1}\right)$, the density $\left(p_{k}\right)$ is of discrete phase type (DPH). Here $\boldsymbol{T}$ is an $m \times n$ substochastic matrix such that $\boldsymbol{T e}_{m}+\boldsymbol{T}^{0}=\boldsymbol{e}_{m}$ and $(\boldsymbol{I}-\boldsymbol{T})$ is non-singular, where $I$ denotes the identity matrix. The density of the time until absorption is given by

$$
\begin{aligned}
& p_{0}=\alpha_{m+1} \\
& p_{k}=\alpha \boldsymbol{T}^{k-1} \boldsymbol{T}^{0} \quad \text { for } k \geqq 1 .
\end{aligned}
$$

The mean of the distribution is $\mu_{p}=\boldsymbol{\alpha}(I-T)^{-1} \boldsymbol{e}_{m}$.

\section{Preservation of phase-type distribution under mixed homogeneous Poisson shock model}

Let $\mathcal{N}$ be a mixture of homogeneous Poisson processes. Then $\bar{H}(t)$ is given by

$$
\bar{H}(t)=\int_{0}^{\infty} \sum_{k=0}^{\infty} \frac{e^{-\lambda t}(\lambda t)^{k}}{k !} \bar{P}_{k} d G(\lambda)
$$

where $\lambda$ is a random variable with cumulative distribution function (c.d.f.) $G($.$) .$

Theorem 3.1. The phase-type distribution is preserved by the transformation (3.1) when $G($.$) is a distribution with finite support.$

Proof. Supposing $\left\{p_{k}\right\}$ has DPH distribution with representation $(\boldsymbol{\alpha}, \boldsymbol{T})$, the survival probabilities $\bar{P}_{k}$ are given by

$$
\bar{P}_{k}=\sum_{n=k+1}^{\infty} \boldsymbol{\alpha} \boldsymbol{T}^{n-1} \boldsymbol{T}^{0}
$$

Since $\boldsymbol{T}$ is finite, $\boldsymbol{T}^{n} \rightarrow \mathbf{0}$ elementwise as $n \rightarrow \infty$ and $\boldsymbol{T} \boldsymbol{e}_{m}+\boldsymbol{T}^{0}=\boldsymbol{e}_{m}$, it is easily verified that

$$
\bar{P}_{k}=\alpha T^{k} \boldsymbol{e}_{m}
$$

From (3.1) and (3.2), it follows immediately that

$$
\bar{H}(t)=\int_{0}^{\infty} \boldsymbol{\alpha} \exp [\lambda(\boldsymbol{T}-\boldsymbol{I}) t] \boldsymbol{e}_{m} d G(\lambda) .
$$


Since the mixing density $G($.$) has finite support, say, \left\{\lambda_{1}, \lambda_{2}, \cdots, \lambda_{k}\right\}$ with mixing density $\left\{q_{1}, q_{2}, \cdots, q_{k}\right\}$, the right-hand side of (3.2) being a finite mixture of phase-type survival functions with representations $\left(\alpha, \lambda_{i}(T-I)\right), i=1,2, \cdots, k$, is again the survival function of a phase-type distribution (see Neuts (1981)). Also the representation of $H(t)$ is $\left(\alpha^{*}, T^{*}\right)$, where $\boldsymbol{\alpha}^{*}=\left(q_{1} \boldsymbol{\alpha}, q_{2} \boldsymbol{\alpha}, \cdots, q_{k} \boldsymbol{\alpha}\right)$ is a row vector with $k m$ components and $\boldsymbol{T}^{*}=$ $\operatorname{diag}\left(\lambda_{1}(T-I), \cdots, \lambda_{k}(T-I)\right)$ is a $k m \times k m$ block-diagonal matrix.

Remark 3.1. The mean of the distribution $H(t)$ is given by $\mu_{H}=-\alpha^{*}\left(T^{*}\right)^{-1} e_{m k}$. After some simple algebra, $\mu_{H}=\sum_{i=1}^{k}\left(q_{i} / \lambda_{i}\right) \mu_{p}$ where $\mu_{p}=\alpha(I-T)^{-1} e_{m}$ denotes the mean of $\left\{p_{k}\right\}$. In particular, if the shocks arrive according to a homogeneous Poisson process with parameter $\lambda$ and the shock probabilities $\left\{p_{k}\right\}$ have DPH distribution with mean $\mu_{p}$, then the life distribution of the device $H(t)$ is $\mathrm{CPH}$ with mean $\mu_{H}=(1 / \lambda) \mu_{p}$.

\section{Acknowledgement}

The authors are grateful to the referee for suggesting improvements in the presentation of the paper.

\section{References}

A-Hameed, M. S. And Proschan, F. (1973) Nonstationary shock models. Stoch. Proc. Appl. 1, 383-404.

A-Hameed, M. S. And Proschan, F. (1975) Shock models with underlying birth process. J. Appl. Prob. 12, 18-28.

Block, H. W. and Savits, T. H. (1978) Shock models with NBUE survival. J. Appl. Prob. 15, 621-628.

Esary, J. D., Marshall, A. W. and Proschan, F. (1973) Shock models and wear processes. Ann. Prob. 1, 627-649.

KLEFSJÖ, B. (1981) HNBUE survival under some shock models. Scand. J. Statist. 8, 39-47.

Neuts, M. F. (1981) Matrix-Geometric Solutions in Stochastic Models-An Algorithmic Approach. The Johns Hopkins University Press, Baltimore. 